\title{
ASSOCIATIONS AND COMMUNITIES OF CEREAL CULTIVATIONS OF THE ŁUKOWSKA PLAIN PART II. ASSOCIATIONS OF HEAVY SOILS
}

\author{
Zofia Rzymowska, Teresa Skrajna \\ Department of Agricultural Ecology of the Siedlce University of Nature Sciences and Humanities \\ B. Prusa 14, 08-110 Siedlce, Poland \\ e-mail: zrzymowska@wp.pl
}

Received: 27.06.2013

Abstract

The paper is the second part of the work examining segetal communities of the Łuków Plain. It presents a description of phytocenoses establishing in cereal crops on heavy soils. Only Vicietum tetraspermae were found in these habitats. Neither patches of Consolido-Brometum nor communities representing the alliance Caucalidion lappulae were encountered. Floristic and ecological diversity of Vicietum tetraspermae was due to 8 syntaxonomic units distinguished in the study area: 3 subassociations (Vicietum tetraspermae typicum, V. t odontitetosum and $V . t$ consolidetosum) and 6 variants within Vicietum tertaspermae typicum. Patches of Vicietum tetraspermae typicum were the most frequent in the study area whereas the remaining sub-alliances were much more rare. Of particular interest were species-poor patches of Vicietum tetraspermae typicum dominated by Vicia grandiflora found in the Łuków area which are indicative of the degradation of this alliance.

Key words: weed communities, cereal cultivations, heavy soils, Vicietum tetraspermae, Consolido-Brometum

\section{INTRODUCTION}

The paper is the second part of the work on weed communities establishing in winter cereals grown in the Łuków Plain (Równina Łukowska) region [1]. The first part, which also contains the description of the study area and methodology, presents the description of the following associations developing on light soils: Arnoserido-Scleranthetum, Papaveretum argemones, Vicietum tetraspermae scleranthetosum and Vicietum tetraspermae typicum, Rhinanthus serotinus variant.

The objective of this work is to present the structure, floristic composition and syntaxonomic diversity of Vicietum tetraspermae establishing in fertile habitats of the Łuków Plain.

\section{RESULTS}

Systematics of the identified cereal associations Class: Stellarietea mediae Tx., Lohm. et Prsg. 1950

Order: Centauretalia cyani R. Tx. 1950

Alliance: Aperion spicae-venti R. Tx. et J. Tx. 1960

1. Association: Vicietum tetraspermae ( $\mathrm{K} \mathrm{r}$ u s e m. et V li e g. 1939) Kornaś 1950

a. Subassociation: Vicietum tetraspermae typicum

- typical variant

- variant with Vicia grandiflora

- variant with Juncus bufonius

- variant with Oxalis fontana

- variant with participation of characteristic species of Panico-Setarion

- variant with participation of characteristic species of Polygono-Chenopodion polyspermi

b. Subassociation: Vicietum tetraspermae odontitetosum

c. Subassociation: Vicietum tetraspermae consolidetosum

A total of 8 syntaxonomic units which reflect the floristic and ecological diversity of Vicietum tetraspermae were determined on the heavy soils of the Łuków Plain area. Moreover, the following 3 subassociations of this association were found (with their variants): Vicietum tetraspermae typicum, Vicietum tetraspermae odontitetosum and Vicietum tetraspermae consolidetosum.

Vicietum tetraspermae typicum was described on the basis of 109 phytosociological relevés. Floristic diversity within the sub-association, which reflects the habitat variation and agrotechnology applied, resulted 
in the following 6 variants: typical, with Vicia grandiflora, with Juncus bufonius, with Oxalis fontana, with species of Panico-Setarion and with species of Polygono-Chenopodion polyspermi. Typical patches of Vicietum tetraspermae typicum (42 phytosociological relevés) were the most popular (Table 1). They established on soils developed from light and heavy loamy sand or silt formations with an underlying layer of light or medium loam. The patches were the floristically poorest communities on heavy soils. Although a total of 78 species were found, the average number of species per relevé, only 14 , was small (8-22) because of a high share of sporadic species (78\%).

Vicia tetrasperma, the characteristic species of the association, was one of the weeds which were dominant in the analysed patches. Bromus secalinus was much rarer in these phytocenoses although with quite high cover. Vicia villosa more frequently accompanied this association and its cover was higher compared with other cereal phytocenoses. On the contrary, Polygonum lapathifolium subsp. pallidum was completely absent. Apart from Vicia terasperma, Apera spica-venti and Vicia hirsuta dominated in typical patches of this sub-association. Moreover, locally, the following species were frequently found: Matricaria maritima subsp. inodora, Anthemis arvensis and Centaurea cyanus with high cover, and Myosotis arvensis and Viola arvensis with lower cover.

Locally, patches of Vicietum tetraspermae typicum dominated by Vicia grandiflora, which were described as a variant with this species, were encountered on the western outskirts of Łuków and along the road to Stoczek Łukowski. The variant was described based on 11 phytosociological relevés in which only 56 species were found (Table 1). The number of species per relevé ranged between 14 and 25, 19 on average. The weed cover in the analysed patches was very high. Apart from the above-mentioned Vicia grandiflora, the phytocenoses had a high share of Apera spica-venti and Centaurea cyanus. Moreover, Vicia villosa and Vicia tetrasperma were a permanent component with high cover, as well as Viola arvensis and Myosotis arvensis, whose cover was lower, however. Other frequent species, with locally higher cover, included: Anthemis arvensis, Stellaria media, Matricaria maritima subsp. inodora, Veronica arvensis and Geranium pusillum. These phytocenoses established on leached brown soils and lessive soils originating from loams or light and heavy loamy sands with an underlying layer of light loam, classified as a very good rye complex, good rye complex or good wheat complex of agricultural land suitability. They were very disturbed habitats located close to buildings and a railway line, within the town limits or in the immediate proximity of the town.

The wet variant of Vicietum tetraspermae typicum was described on the basis of 25 plant patches establishing on lessive soils and degraded black earths representing rye complexes (5 and 4), a poor wheat complex (3) and cereal-fodder complexes (8 and 9). A total of 115 species occurred in these phytocenoses (Table 1). They were floristically abundant communities with 12 to 35 species per relevé, 26 on average. Of the characteristic species, Vicia tetrasperma occurred on a large scale in the analysed patches whereas other species were less frequent and their cover was lower. The patches were also dominated by Apera spica-venti and characterised by a large group of hygrophilous species, with a permanent occurrence of Juncus bufonius (high cover) and Gnaphalium uliginosum (lower cover). Other species, for example: Mentha arvensis, Polygonum hydropiper, Plantago intermedia, Gypsophila muralis, were less frequently found. Other numerous species included: Vicia hirsuta (high cover), Vicia angustifolia, Stellaria media, Myosotis arvensis, Viola arvensis, Veronica arvensis and locally Matricaria maritima subsp. inodora.

Vicietum tetraspermae typicum, the variant with Oxalis fontana, was distinguished based on 11 phytosociological relevés (Table 1). Such phytocenoses established mainly in spring cereals in moderately fertile habitats which were sufficiently or excessively wet, with near-neutral $\mathrm{pH}$. Their soils were as follows: lessive soils, leached brown soils as well as degraded and proper black earths classified as cereal-fodder complexes (8 and 9) and a very good rye complex (4). The phytocenoses were floristically quite rich, with an average number of species per relevé of 24 (15-33) and a total of only 73 species. Vicia tetrasperma was a permanent component of these communities but its cover was not high whereas Vicia villosa was found only occasionally. More frequent species included Bromus secalinus and Polygonum lapathifolium subsp. pallidum. Apart from Oxalis fontana, other permanent species included Vicia angustifolia and Viola arvensis whereas Myosotis arvensis, Fallopia convolvulus, Anthemis arvensis and Matricaria maritima subsp. inodora were frequent components. By contrast, Apera spica-venti was a relatively rare species. A numerous group of hygrophilous species occurring in these habitats, Mentha arvensis and Ranunculus repens being the most popular, is indicative of an excess of moisture. 
Table 1

Vicietum tetraspermae (Krusem et Vlieg. 1939). Part. I

\begin{tabular}{|c|c|c|c|c|c|c|c|c|}
\hline Association & \multicolumn{8}{|c|}{ Vicietum tetraspermae } \\
\hline Subassociation & \multicolumn{8}{|c|}{ typicum } \\
\hline Variant & \multicolumn{2}{|c|}{ typical } & \multicolumn{2}{|c|}{$\begin{array}{c}\text { with Vicia } \\
\text { grandiflora }\end{array}$} & \multicolumn{2}{|c|}{$\begin{array}{c}\text { with Juncus } \\
\text { bufonius }\end{array}$} & \multicolumn{2}{|c|}{$\begin{array}{l}\text { with Oxalis } \\
\text { fontana }\end{array}$} \\
\hline Number of relevés & \multicolumn{2}{|c|}{42} & \multicolumn{2}{|c|}{11} & \multicolumn{2}{|c|}{25} & \multicolumn{2}{|c|}{11} \\
\hline \multirow[b]{3}{*}{ Soil unit } & \multicolumn{2}{|c|}{5,4} & \multicolumn{2}{|c|}{$5,4,2$} & \multicolumn{2}{|c|}{$5,4,3,8,9$} & \multicolumn{2}{|c|}{$4,8,9$} \\
\hline & \multicolumn{2}{|c|}{$\mathrm{A}, \mathrm{Bw}, \mathrm{D}$} & \multicolumn{2}{|c|}{$\mathrm{A}, \mathrm{Bw}$} & \multicolumn{2}{|c|}{$\mathrm{A}, \mathrm{Dz}, \mathrm{D}$} & \multicolumn{2}{|c|}{$\mathrm{A}, \mathrm{Bw}, \mathrm{Dz}, \mathrm{D}$} \\
\hline & \multicolumn{2}{|c|}{$\begin{array}{l}\text { pgl; pgl:gl; pgl.gl; } \\
\text { pglp; pglp:ps; pglp:gs; } \\
\text { pgm.ps; pgm:gs; } \\
\text { pgmp:ps; pgmp:gl; } \\
\text { płz:ps; płz:gl; płz:gs }\end{array}$} & \multicolumn{2}{|c|}{$\begin{array}{l}\text { pgl:gl; pgm. } \\
\text { gl; gl }\end{array}$} & \multicolumn{2}{|c|}{$\begin{array}{l}\text { pgl.pl.płz; pgl. } \\
\text { ps; pgl:gl; pgl. } \\
\text { gs; pgm.ps; } \\
\text { pgm; pgm:glp; } \\
\text { pgm.gl; płz; glp }\end{array}$} & \multicolumn{2}{|c|}{$\begin{array}{l}\text { pgl.ps; pgl:gl; } \\
\text { pgm:ps; pgm. } \\
\text { gl; pgmp; płz. } \\
\text { gs; glp }\end{array}$} \\
\hline Range of number of species in relevés & \multicolumn{2}{|c|}{$8-22$} & \multicolumn{2}{|c|}{$14-25$} & \multicolumn{2}{|c|}{$12-35$} & \multicolumn{2}{|c|}{$15-33$} \\
\hline Mean number of species in relevé & \multicolumn{2}{|c|}{14} & \multicolumn{2}{|c|}{19} & \multicolumn{2}{|c|}{26} & \multicolumn{2}{|c|}{24} \\
\hline \multirow[t]{2}{*}{ Number of columns } & \multicolumn{2}{|c|}{1} & \multicolumn{2}{|c|}{2} & \multicolumn{2}{|c|}{3} & \multicolumn{2}{|c|}{4} \\
\hline & $\mathrm{S}$ & $\mathrm{W}$ & $S$ & $\mathrm{~W}$ & $\mathrm{~S}$ & $\mathrm{~W}$ & S & W \\
\hline \multicolumn{9}{|l|}{ I.Ch.D. Vicietum tetraspermae } \\
\hline Vicia tetrasperma & $\mathrm{V}$ & 806 & $\mathrm{~V}$ & 350 & $\mathrm{~V}$ & 718 & $\mathrm{~V}$ & 395 \\
\hline Vicia villosa & III & 255 & $\mathrm{~V}$ & 377 & II & 130 & I & 50 \\
\hline Bromus secalinus & II & 201 & I & 45 & I & 28 & II & 27 \\
\hline Polygonum lapathifolium subsp. pallidum & & & & & II & 24 & II & 100 \\
\hline
\end{tabular}

\section{D. var. z Vicia grandiflora}

Vicia grandiflora V 1100

\section{D. var. z Oxalis fontana}

\begin{tabular}{|c|c|c|c|c|c|c|c|c|}
\hline Oxalis fontana & & & & & I & 8 & $\mathrm{~V}$ & 505 \\
\hline \multicolumn{9}{|l|}{ IV. D. var. z Juncus bufonius } \\
\hline Juncus bufonius & & & I & 9 & $\mathrm{~V}$ & 712 & III & 55 \\
\hline Gnaphalium uliginosum & & & & & $\mathrm{V}$ & 148 & III & 55 \\
\hline Gypsophila muralis & I & 5 & & & III & 88 & II & 36 \\
\hline Plantago intermedia & I & 14 & & & III & 116 & II & 27 \\
\hline Mentha arvensis & & & & & III & 166 & II & 486 \\
\hline Polygonum hydropiper & & & & & III & 162 & $\mathrm{I}$ & 18 \\
\hline Ranunculus repens & $\mathrm{I}$ & 5 & & & II & 28 & II & 186 \\
\hline Potentilla anserina & & & & & II & 48 & II & 27 \\
\hline Spergularia rubra & & & & & II & 28 & $\mathrm{I}$ & 18 \\
\hline Sagina procumbens & & & & & II & 48 & I & 18 \\
\hline Stachys palustris & & & & & II & 40 & $\mathrm{I}$ & 9 \\
\hline \multicolumn{9}{|c|}{ V. Ch. Aperion spicae-venti, Centauretalia cyani } \\
\hline Apera spica-venti & $\mathrm{V}$ & 1099 & $\mathrm{~V}$ & 764 & IV & 1098 & III & 500 \\
\hline Vicia hirsuta & $\mathrm{V}$ & 808 & II & 36 & IV & 580 & III & 390 \\
\hline Centaurea cyanus & IV & 320 & $\mathrm{~V}$ & 609 & III & 60 & II & 36 \\
\hline Vicia angustifolia & III & 95 & III & 45 & IV & 108 & $\mathrm{~V}$ & 82 \\
\hline Anthemis arvensis & IV & 320 & IV & 222 & III & 126 & IV & 64 \\
\hline Matricaria maritima subsp. inodora & IV & 360 & IV & 145 & II & 410 & IV & 64 \\
\hline Agrostemma githago & I & 54 & II & 72 & $\mathrm{I}$ & 20 & & \\
\hline Rhinanthus serotinus & & & II & 64 & & & $\mathrm{I}$ & 9 \\
\hline
\end{tabular}




\begin{tabular}{|c|c|c|c|c|c|c|c|c|}
\hline \multicolumn{9}{|c|}{ VI. Ch. Stellarietea mediae } \\
\hline Viola arvensis & $\mathrm{V}$ & 112 & $\mathrm{~V}$ & 209 & IV & 72 & V & 118 \\
\hline Stellaria media & III & 45 & IV & 160 & IV & 182 & II & 109 \\
\hline Myosotis arvensis & IV & 199 & $\mathrm{~V}$ & 136 & IV & 132 & IV & 64 \\
\hline Chenopodium album & I & 24 & III & 45 & III & 64 & III & 45 \\
\hline Fallopia convolvulus & II & 26 & II & 27 & I & 20 & IV & 109 \\
\hline Capsella bursa-pastoris & III & 52 & III & 54 & III & 56 & I & 9 \\
\hline Sonchus arvensis & I & 7 & I & 9 & II & 56 & III & 45 \\
\hline Conyza canadensis & II & 89 & I & 9 & II & 40 & III & 45 \\
\hline Polygonum aviculare & I & 7 & & & III & 52 & I & 55 \\
\hline Geranium pusillum & I & 7 & III & 195 & I & 16 & & \\
\hline Galeopsis tetrahit & I & 2 & & & II & 32 & II & 27 \\
\hline Spergula arvensis & & & & & I & 12 & II & 177 \\
\hline Anagallis arvensis & I & 19 & & & II & 98 & I & 9 \\
\hline Rumex crispus & I & 5 & & & II & 28 & I & 18 \\
\hline Descurainia sophia & & & II & 64 & & & $\mathrm{I}$ & 0 \\
\hline \multicolumn{9}{|c|}{ VII. Accompanying species } \\
\hline Veronica arvensis & I & 17 & IV & 64 & IV & 84 & II & 36 \\
\hline Cirsium arvense & II & 45 & II & 64 & III & 112 & III & 45 \\
\hline Galium aparine & III & 198 & II & 223 & II & 112 & II & 214 \\
\hline Equisetum arvense & II & 87 & II & 27 & II & 44 & III & 195 \\
\hline Convolvulus arvensis & II & 36 & I & 18 & I & 8 & III & 82 \\
\hline Elymus repens & II & 43 & II & 36 & II & 84 & II & 64 \\
\hline Galeopsis bifida & & & II & 27 & II & 8 & II & 27 \\
\hline Achillea millefolium & I & 7 & I & 9 & II & 40 & II & 27 \\
\hline Rumex acetosella & I & 2 & I & 9 & II & 24 & II & 73 \\
\hline Cerastium holosteoides & I & 14 & II & 27 & II & 32 & I & 18 \\
\hline Arenaria serpyllifolia & I & 46 & II & 64 & I & 4 & I & 9 \\
\hline Artemisia vulgaris & I & 14 & II & 27 & I & 20 & I & 18 \\
\hline Trifolium repens & I & 5 & I & 9 & II & 24 & I & 9 \\
\hline Erodium cicutarium & I & 7 & II & 27 & & & I & 18 \\
\hline Medicago falcata & I & 5 & II & 27 & & & & \\
\hline Taraxacum officinale & I & 7 & & & II & 20 & I & 9 \\
\hline \multicolumn{9}{|c|}{ 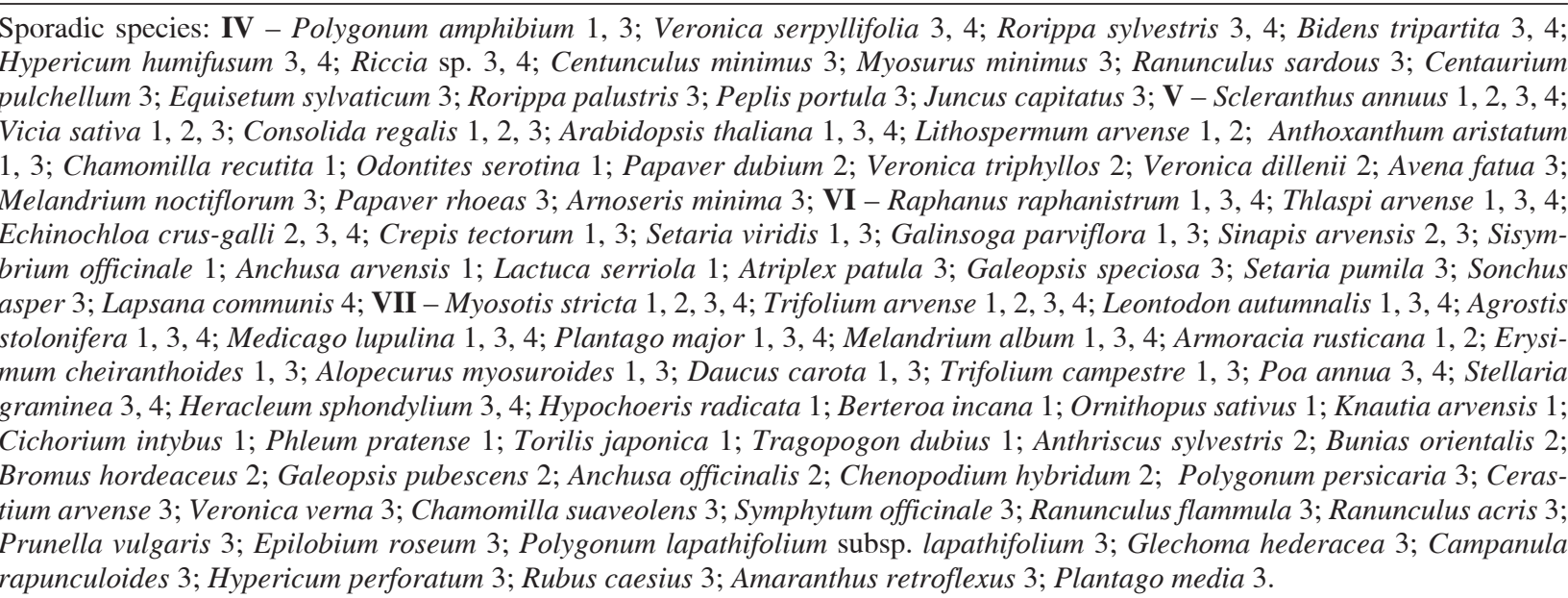 } \\
\hline
\end{tabular}


Impoverished patches of Vicietum tetraspermae typicum were found in spring cereals with a low share of characteristic species of this association, with local mass occurrence of weeds which typically accompany tuber and root crops. Patches with characteristic species of Panico-Setarion established on the soils of very good rye complex as well as cereal-fodder complexes, Echinochloa crus-galli being a permanent component with high cover. Other Panico-Setarion species were found only occasionally, apart from $\mathrm{Se}$ taria pumila which was more frequent (Table 2). In addition to Echinochloa crus-galli, the phytocenoses were dominated by hygrophilous species, which is indicative of excessive moisture of these habitats. Juncus bufonius and Polygonum hydropiper had the highest cover. Other popular species included for example: Gnaphalium uliginosum, Plantago intermedia, Mentha arvensis, Stachys palustris, Rorippa sylvestris, Bidens tripartita and Potentilla anserina. Apera spica-venti and Vicia hirsuta were quite frequent and had high cover, too. The phytocenoses were floristically rich as the number of species per relevé ranged between 24 and 37, 28 on average.

More fertile habitats of the good wheat complex and cereal-fodder complexes had patches of Vicietum tetraspermae typicum with a share of characteristic species of Polygono-Chenopodion polyspermi. They were floristically rich phytocenoses with 28 species per relevé, on average (the range: 22-43) (Table 2). Just like in the above-mentioned phytocenoses, these communities had a low share of characteristic species of the association. However, there was found a numerous group of species which are indicators of substantial fertility of these habitats. They included: Veronica persica, Lamium purpureum, Sonchus asper and Veronica agrestis and other sporadic species. The following hygrophilous species were also popular in these patches: Juncus bufonius, Plantago intermedia, Gnaphalium uliginosum, Mentha arvensis, Potentilla anserina and others. Also, Vicia hirsuta, Viola arvensis and Anagallis arvensis were frequent components of these phytocenoses although in other cereal phytocenoses they were only occasionally found. Compared with other phytocenoses, Apera spica-venti was much more rare and had lower cover. What was interesting was the presence of rare species of carbonate and fertile habitats, such as: Galeopsis speciosa,
Aethusa cynapium, Atriplex patula, Geranium dissectum, Lamium amplexicaule, Veronica polita, Chenopodium polyspermum and Euphorbia helioscopia, which, as a rule, were not found in other cereal communities in this area

Vicietum tetraspermae odontitetosum was described based on 13 plant patches. It was found on various soils classified as rye complexes (4 and 5), a poor wheat complex (3) and cereal-fodder complexes (8 and 9) (Table 2). Vicia tetrasperma had the highest cover in patches of this sub-association. Vicia villosa was also quite frequent whereas Bromus secalinus and Polygonum lapathifolium subsp. pallidum were only occasionally found. An occurrence of Odontites serotina and Rhinanthus serotinus distinguished these phytocenoses. In patches of this sub-association, Apera spica-venti had the highest cover of all the syntaxons found. Vicia hirsuta and Galium aparine were also numerous, although the latter was found only locally. The following hygrophilous species were components of these phytocenoses: Juncus bufonius (high cover) Gnaphalium uliginosum, Mentha arvensis, Stachys palustris, Gypsophila muralis, Polygonum hydropiper and Ranunculus sardous (locally high cover).

Vicietum tetraspermae consolidetosum established on soils richer in calcium carbonate which belonged to very good and good rye complexes as well as good wheat and good cereal-fodder complexes (Table 2). Of the association's characteristic species, only Vicia tetrasperma was the permanent component of the weed flora with high cover whereas the remaining species were quite infrequent. Consolida regalis was the most popular of the species which distinguish this sub-association whereas Papaver rhoeas and Lithospermum arvense were more rare and had lower cover. Centaurea cyanus was a permanent component of these phytocenoses with the greatest cover. Frequent weeds were: Apera spica-venti and Cirsium arvense, both with high cover, and Viola arvensis, Myosotis arvensis, Vicia angustifolia and Veronica arvensis, all with lower cover. Agrostemma githago occurred locally with higher cover, too. Rare carbonate species included only Melandrium noctiflorum, Anagallis arvensis, Geranium dissectum and Anchusa arvensis. Moreover, the following nitrophilous species were found: Galium aparine, Matricaria maritima subsp. inodora, Sonchus asper and Lamium purpureum. 
Table 2

Vicietum tetraspermae (Krusem. et Vlieg. 1939). Part. II

\begin{tabular}{|c|c|c|c|c|c|c|c|c|}
\hline Association & \multicolumn{8}{|c|}{ Vicietum tetraspermae } \\
\hline Subassociation & \multicolumn{4}{|c|}{ typicum } & \multicolumn{2}{|c|}{ odontitetosum } & \multicolumn{2}{|c|}{ consolidetosum } \\
\hline Variant & \multicolumn{2}{|c|}{$\begin{array}{l}\text { with Panico- } \\
\text { Setarion }\end{array}$} & \multicolumn{2}{|c|}{$\begin{array}{l}\text { with Polygono- } \\
\text { Chenopodion }\end{array}$} & \multicolumn{2}{|c|}{ typical } & \multicolumn{2}{|c|}{ typical } \\
\hline Number of relevés & \multicolumn{2}{|c|}{10} & \multicolumn{2}{|c|}{10} & \multicolumn{2}{|c|}{13} & \multicolumn{2}{|c|}{13} \\
\hline \multirow[b]{3}{*}{ Soil unit } & \multicolumn{2}{|c|}{$2,8,9$} & \multicolumn{2}{|c|}{$2,4,8$} & \multicolumn{2}{|c|}{$5,4,2,8,9$} & \multicolumn{2}{|c|}{$5,4,2,8$} \\
\hline & \multicolumn{2}{|c|}{$\mathrm{A}, \mathrm{M}, \mathrm{Dz}, \mathrm{D}$} & \multicolumn{2}{|c|}{$\mathrm{A}, \mathrm{Bw}, \mathrm{Dz}, \mathrm{D}$} & \multicolumn{2}{|c|}{$\mathrm{A}, \mathrm{Bw}, \mathrm{D}$} & \multicolumn{2}{|c|}{$\mathrm{A}, \mathrm{Bw}, \mathrm{Dz}$} \\
\hline & \multicolumn{2}{|c|}{$\begin{array}{l}\text { pgl.ps; pgl:gl; } \\
\text { pgl.płz; pgm.gl; } \\
\text { płz; glp; gl }\end{array}$} & \multicolumn{2}{|c|}{$\begin{array}{l}\text { pgl:gl; pgl.gl; } \\
\text { płz.gl; pglp }\end{array}$} & \multicolumn{2}{|c|}{$\begin{array}{l}\text { pgl:ps; pgl:gl; } \\
\text { pgl.ps:gs; } \\
\text { pgm:gl; płz }\end{array}$} & \multicolumn{2}{|c|}{$\begin{array}{l}\text { pgl; pglp; pgl:gl; } \\
\text { pgmp:ps; pgmp:gl; } \\
\text { pgm.pgl:gl; pgmp; } \\
\text { pgmp:gs; glp; gs.gc }\end{array}$} \\
\hline Range of number of species in relevés & \multicolumn{2}{|c|}{$24-37$} & \multicolumn{2}{|c|}{$22-43$} & \multicolumn{2}{|c|}{$14-31$} & \multicolumn{2}{|c|}{$10-25$} \\
\hline Mean number of species in relevé & \multicolumn{2}{|c|}{28} & \multicolumn{2}{|c|}{28} & \multicolumn{2}{|c|}{23} & & \\
\hline Number of columns & & & & & & 3 & & \\
\hline & $\mathrm{S}$ & $\mathrm{W}$ & $\mathrm{S}$ & $\mathrm{W}$ & $\mathrm{S}$ & $\mathrm{W}$ & $\mathrm{S}$ & $\mathrm{W}$ \\
\hline I. Ch. D. Vicietum tetraspermae & & & & & & & & \\
\hline Vicia tetrasperma & $\mathrm{V}$ & 220 & V & 260 & $\mathrm{~V}$ & 995 & $\mathrm{~V}$ & 627 \\
\hline Vicia villosa & I & 50 & & & II & 205 & II & 181 \\
\hline Bromus secalinus & II & 30 & II & 40 & I & 10 & II & 62 \\
\hline Polygonum lapathifolium subsp. pallidum & & & II & 30 & I & 10 & I & 8 \\
\hline II. Ch. Panico-Setarion & & & & & & & & \\
\hline Echinochloa crus-galli & $\mathrm{V}$ & 660 & I & 60 & & & I & 8 \\
\hline Setaria pumila & II & 70 & & & & & & \\
\hline III. Ch. Polygono-Chenopodion & & & & & & & & \\
\hline Veronica persica & I & 20 & IV & 190 & & & & \\
\hline Lamium purpureum & & & III & 50 & & & I & 8 \\
\hline Sonchus asper & I & 20 & II & 30 & & & I & 8 \\
\hline Lapsana communis & & & I & 10 & & & II & 15 \\
\hline Veronica agrestis & & & II & 40 & & & & \\
\hline
\end{tabular}

\section{D. V t. odontitetosum}

Odonites serotina

Rhinanthus serotinus

$\begin{array}{cccc}\text { V } & 590 & \text { I } & 142 \\ \text { III } & 590 & \text { I } & 142\end{array}$

\section{D. V t. consolidetosum}

Consolida regalis

Papaver rhoeas

II $\quad 150$

Lithospermum arvense

II 92

\section{D. var. z Juncus bufonius}

Juncus bufonius

Polygonum hydropiper

Gnaphalium uliginosum

Plantago intermedia

Mentha arvensis

Stachys palustris

$\begin{array}{lccccccc}\text { IV } & 935 & \text { IV } & 475 & \text { IV } & 720 & \text { I } & 8 \\ \text { V } & 630 & \text { II } & 30 & \text { III } & 90 & & \\ \text { V } & 130 & \text { IV } & 120 & \text { IV } & 150 & \text { I } & 15 \\ \text { IV } & 285 & \text { IV } & 160 & \text { II } & 30 & & \\ \text { III } & 170 & \text { III } & 90 & \text { III } & 295 & & \\ \text { III } & 100 & \text { II } & 80 & \text { III } & 215 & & \end{array}$




\begin{tabular}{|c|c|c|c|c|c|c|c|c|}
\hline Gypsophila muralis & I & 10 & I & 20 & III & 90 & I & 8 \\
\hline Rorippa sylvestris & III & 50 & I & 10 & I & 10 & & \\
\hline Potentilla anserina & II & 195 & II & 80 & II & 70 & & \\
\hline Ranunculus sardous & I & 50 & & & II & 462 & & \\
\hline Bidens tripartita & II & 195 & I & 60 & I & 10 & & \\
\hline Ranunculus repens & II & 30 & I & 60 & I & 50 & & \\
\hline Sagina procumbens & II & 30 & I & 10 & I & 10 & & \\
\hline Spergularia rubra & II & 40 & & & I & 10 & & \\
\hline Polygonum amphibium & I & 50 & II & 40 & & & I & 8 \\
\hline Rorippa palustris & II & 70 & I & 10 & & & & \\
\hline \multicolumn{9}{|c|}{ VII. Ch. Aperion spicae-venti, Centauretalia cyani } \\
\hline Apera spica-venti & IV & 695 & III & 595 & IV & 1700 & IV & 581 \\
\hline Centaurea cyanus & II & 30 & II & 30 & $\mathrm{~V}$ & 285 & $\mathrm{~V}$ & 400 \\
\hline Vicia hirsuta & III & 465 & IV & 245 & $\mathrm{~V}$ & 1000 & III & 335 \\
\hline Matricaria maritima subsp. inodora & I & 225 & III & 215 & IV & 355 & $\mathrm{~V}$ & 342 \\
\hline Vicia angustifolia & III & 100 & II & 40 & IV & 120 & IV & 92 \\
\hline Anthemis arvensis & II & 30 & II & 30 & III & 505 & II & 62 \\
\hline Avena fatua & II & 70 & & & & & I & 8 \\
\hline Scleranthus annuus & I & 10 & I & 10 & II & 70 & I & 15 \\
\hline Agrostemma githago & & & & & & & II & 431 \\
\hline \multicolumn{9}{|l|}{ VIII. Ch. Stellarietea mediae } \\
\hline Viola arvensis & II & 30 & IV & 110 & $\mathrm{~V}$ & 120 & IV & 131 \\
\hline Stellaria media & IV & 160 & III & 265 & II & 70 & III & 38 \\
\hline Myosotis arvensis & III & 50 & III & 60 & IV & 110 & IV & 92 \\
\hline Anagallis arvensis & II & 30 & IV & 110 & II & 30 & I & 8 \\
\hline Spergula arvensis & IV & 120 & & & I & 20 & & \\
\hline Chenopodium album & III & 170 & III & 90 & I & 10 & $\mathrm{I}$ & 15 \\
\hline Fallopia convolvulus & I & 10 & III & 60 & II & 20 & III & 46 \\
\hline Capsella bursa-pastoris & II & 30 & II & 30 & II & 30 & III & 77 \\
\hline Polygonum aviculare & & & II & 40 & I & 10 & I & 15 \\
\hline Sonchus arvensis & III & 90 & III & 90 & I & 10 & & \\
\hline Conyza canadensis & I & 10 & I & 10 & II & 70 & II & 62 \\
\hline Rumex crispus & II & 20 & I & 20 & I & 0 & & \\
\hline Galeopsis tetrahit & II & 120 & I & 20 & & & & \\
\hline Galeopsis speciosa & & & II & 70 & & & & \\
\hline Anchusa arvensis & & & & & & & II & 31 \\
\hline \multicolumn{9}{|l|}{ IX. Accompanying species } \\
\hline Cirsium arvense & III & 140 & III & 60 & IV & 160 & IV & 92 \\
\hline Veronica arvensis & III & 60 & IV & 70 & III & 50 & IV & 62 \\
\hline Galium aparine & I & 20 & III & 180 & II & 235 & IV & 219 \\
\hline Elymus repens & III & 180 & III & 170 & I & 10 & I & 15 \\
\hline Equisetum arvense & II & 30 & III & 50 & II & 30 & $\mathrm{I}$ & 15 \\
\hline Convolvulus arvensis & I & 10 & II & 40 & II & 80 & III & 69 \\
\hline Rumex acetosella & I & 20 & I & 10 & III & 100 & I & 8 \\
\hline Polygonum persicaria & III & 50 & II & 40 & I & 10 & & \\
\hline Medicago lupulina & I & 20 & III & 50 & & & II & 23 \\
\hline Artemisia vulgaris & III & 20 & $\mathrm{I}$ & 10 & & & II & 23 \\
\hline
\end{tabular}




\begin{tabular}{lcccccccc}
\hline Plantago major & I & 10 & III & 40 & & & I & 8 \\
Cerastium holosteoides & I & 10 & II & 30 & II & 10 & I & 15 \\
Trifolium repens & I & 0 & II & 20 & II & 30 & I & 8 \\
Taraxacum officinale & II & 10 & II & 30 & I & 20 & I & 15 \\
Agrostis stolonifera & I & 10 & I & 10 & II & 40 & & \\
Achillea millefolium & II & 20 & I & 10 & I & 10 & I & 8 \\
Trifolium arvense & I & 10 & & & I & 20 & II & 62 \\
Poa annua & II & 110 & I & 20 & & & & \\
Trifolium campestre & II & 30 & I & 10 & & & I & \\
Chamomilla suaveolens & II & 70 & I & 20 & & & & \\
\hline
\end{tabular}

Sporadic species: II - Raphanus raphanistrum 1, 2, 3, 4; Setaria viridis 2; III - Galinsoga parviflora 1, 2, 3; Oxalis fontana 1, 2; Sonchus oleraceus 2, 3; Lamium amplexicaule 2, 4; Veronica polita 2; Chenopodium polyspermum 2; Euphorbia helioscopia 2; VI - Centunculus minimus 1, 2, 3, 4; Riccia sp. 1, 2; Equisetum sylvaticum 2, 3; Myosurus minimus 2, 3; Centaurium pulchellum 1; Anthoceros punctatus 2; Phragmites australis 2; Illecebrum verticillatum 2; Veronica serpyllifolia 3; VII - Vicia sativa 2, 3; Arabidopsis thaliana 3, 4; Aethusa cynapium 2; Chamomilla recutita 3; Odontites verna 3; Melandrium noctiflorum 4; VIII Sinapis arvensis 1, 2; Geranium dissectum 2, 4; Thlaspi arvense 3, 4; Atriplex patula 2; Sisymbrium officinale 2; Crepis tectorum 4; IX - Lolium perenne 1, 2, 3; Stellaria graminea 1, 2, 3; Polygonum lapathifolium subsp. lapathifolium 1, 2; Galeopsis bifida 1, 2; Melilotus alba 2, 3; Knautia arvensis 1; Leontodon autumnalis 2; Rumex obtusifolius 2; Galium boreale 2; Cichorium intybus 2; Erophila verna 3; Galeopsis ladanum 3; Medicago falcata 3; Alopecurus myosuroides 3; Trifolium pratense 3; Lotus corniculatus 3; Potentilla collina 3; Festuca ovina 3; Trifolium medium 3; Glechoma hederacea 3; Plantago lanceolata 3; Pimpinella saxifraga 3; Arenaria serpyllifolia 4; Myosotis stricta 4; Urtica dioica 4; Berteroa incana 4; Daucus carota 4.

\section{DISCUSSION}

Vicietum tetraspermae, which is the most widespread association in Poland, was found on heavy soils of the Łuków Plain. It is common in many regions of Poland [2-5] and others, being less frequent only in the north-eastern part of Poland [3, 6]. The habitat and ecological amplitude of the association in terms of soil moisture and nutrient availability is, respectively, quite wide and wide. It is reflected in the local habitat diversity and division of the association into lower syntaxonomic units [2, 3, 5, 7-10] and others. Moreover, the floristic diversity of Vicietum tetraspermae in the area of the Łuków Plain reflects the association's habitat variation. Typical patches of Vicietum tetraspermae were the most popular whereas the wet variant with Juncus bufonius was less frequent.

The variant with Vicia grandiflora indicates a kind of degradation of Vicietum tetraspermae typicum patches in which a mass occurrence of Vicia grandiflo$r a$ leads to impoverishment of floristic and phytocenotic richness of these communities. Such phytocenoses in the area of the Łuków Plain occur locally and only on the outskirts of the town along the road to Stoczek Łukowski. Phytocenoses dominated by Vicia grandiflora have been mentioned by $\mathrm{W}$ a r c holi ń s k a [11, 12], Wójcik [13], Ziaja and Wnuk [14]. The status of this species is ambiguous in Poland. In many regions is it classified as an expansive species $[11,12$, $14,15]$ whereas in others it is a rare species [16-18]. Similar situation has been noticed in the South Podlasie Lowland where Vicia grandiflora is expansive only in some areas. The population of Vicia grandiflora on the outskirts of Łuków revealed expansive tendencies but recent studies by $\mathrm{Ciosek}$ and $\mathrm{Skrzyczy}$ s ska [19] conducted in that region have demonstrated that the species has been withdrawing from these sites.

The carbonate-richest habitats in the study area were covered by patches of Vicietum tetraspermae consolidetosum as no phytocenoses were found representing the association Caucalidion lappulae reported in various regions, in particular the southern and southeastern part of Poland: the Lublin region [20, 21], Zamość area [22], the Polish Jurassic Highland [23], the Przedbórz-Małogoszcz Chain and Poland [24, 25], the Szczerców Valley [26], Sieradz area [27], the Piotrków Plain and Radomsko Hills [28, 29] and the Skierbieszów Landscape Park [30]. Consolido-Brometum, which has been found in the Przemyśl Foothills [13], the Suwałki Lake District [6] and the Skierbiszew National Park [30], was absent in the study area. The core range of Vicietum tetraspermae consolidetosum species was similar to that reported by Węgrzynek [4] for the Silesian Upland, but the phytocenoses in this study were poorer due to lack of rare calcium-loving species from the association Caucalidion lappulae. Species from the aforementioned ecologic group in the area of the Łuków Plain were represented by Avena fatua and Melandrium noctiflorum.

The sub-association Vicietum tetraspermae odontitetosum, which is quite rare in Poland $[9,23,24$, $31,32]$, occurred in the study area.

Communities establishing in spring cereals in the study area can be classified as an impoverished form 
of Vicietum tetraspermae with a high share of species characteristic of Panico-Setarion and Polygono-Chenopodion polyspermi which are typical of tuber and root crops. The characteristic species of the association in these phytocenoses are not frequent but they were classified as communities with Aperion spicae-venti because they contained numerous species characteristic of the alliance and order Centauretalia cyani. Communities in spring cereals which are difficult to classify unambiguously are usually not included in phytosociological works. Sometimes, better developed patches presented in tables accompany phytocenoses of winter cereals where communities are better developed and represent typical patches of cereal associations [2, 24] and others. A different approach has been employed by Kozak [33] who has classified them as tuber and root crop communities, thus stressing that they are more similar to tuber and root rater than cereal communities.

\section{Acknowledgements}

Research supported by the Ministry of Science and Higher Education of Poland as the part of statutory activities of Department of Agricultural Ecology, Siedlce University of Natural Sciences and Humanities.

\section{Author's contributions}

The following declarations about authors' contributions to the research have been made: concept of the study: ZR, ST; field work: ZR, ST; data analysis: ZR, ST; writing of the manuscript: ZR, ST.

\section{REFERENCES}

1. Rzymowska Z, Skrajna T. Associations and communities of the cereal crops of the Łuków Plain. Part. I. Light soil associations. Acta Agrobot. 2011; 64(4): 243-250. http://dx.doi.org/10.5586/aa.2011.066

2. A nioł-Kwiatkowska J. Zbiorowiska segetalne Wału Trzebnickiego. Florystyczno-ekologiczne studium porównawcze. Wyd. Uniw. Wrocławskiego, Pr. Bot. 1990; 46: 1-230. (in Polish)

3. Warcholińska AU. Vicietum tetraspermae w Polsce. Fragm. Flor. Geobot. Ser. Polonica. 1999; 6: 95-117.

4. Węg r z y nek B. Roślinność segetalna Wyżyny Śląskiej. Cz. 2. Zbiorowiska chwastów upraw zbożowych ze związku Aperion spicae-venti. Acta Biol. Siles. 2003; 37(54): 87-119. (in Polish)

5. Wnuk Z. Zbiorowiska segetalne Wyżyny Częstochowskiej na tle zbiorowisk segetalnych Polski. Monogr. Bot. 1989; 71: 1-118. (in Polish)

6. Wójcik Z. Zbiorowiska segetalne Pojezierza Suwalskiego. Fragm. Flor. Geobot. Ser. Polonica. 2000; 7: 167208.
7. Warcholińska AU. Klasyfikacja numeryczna zbiorowisk segetalnych Wzniesień Łódzkich. 1990; pp. $210+$ Tabele. Wyd. Uniw. Łódzkiego, Łódź.

8. Jackowiak B, Chmiel J, Latowski K. Zbiorowiska segetalne zbóż ozimych Wielkopolski. Cz. I. Part. II. Bad. Fizjogr. Pol. Zach., Ser. B. Botanika. 1990; 43: 105123.

9. Skrzyczy ń sk a J. Studia nad florą i zbiorowiskami segetalnymi Wysoczyzny Siedleckiej. Rozp. Nauk. AP Siedlce. 1994 ; 39; pp. 145.

10. Skrzyczyńska J, Rzymowska Z. Zbiorowiska roślinne pól uprawnych Podlaskiego Przełomu Bugu. Cz. I. Zespoły zbożowe. Acta Agrobot. 2005; 58(1): 255-290. http://dx.doi.org/10.5586/aa.2005.028 (in Polish)

11. Warcholińska AU. Współczesne przeobrażenia zbiorowisk segetalnych w środkowej Polsce. Acta Agrobot. 1979; 32(2): 239-269.

12. Wa r cholińs k a A U. Ekspansja Vicia grandiflora Scop. w środkowej Polsce. Zesz. Nauk. ATR Bydgoszcz 196, Roln. 1996; 38: 173-182.

13. Wójcik Z. Zbiorowiska segetalne Pogórza Przemyskiego i jego najbliższego otoczenia. Fragm. Flor. Geobot. Ser. Polonica. 1998; 5:117-164.

14. Zi aja M., Wnuk Z. Vicia grandiflora Scop. w zbiorowiskach segetalnych doliny rzeki Strug w województwie podkarpackim. Fragm. Agron. 2010; 27(3): 167-172.

15. Skrajna T, Skrzyczyńska J, Ługowska M. Segetal communities of cereal crops of the Mazowiecki Landscape Park. Acta Agrobot. 2009; 62(1): 171-186. http://dx.doi.org/10.5586/aa.2009.020

16. Fijałkowski D. Flora roślin naczyniowych Lubelszczyzny. T. 1. Środowisko przyrodnicze Lubelszczyzny. Lubelskie Towarzystwo Przyrodnicze, Lublin, 1994. pp. 389.

17. Fijałkowski D, Nycz B. Zagrożone gatunki roślin segetalnych na Lubelszczyźnie. Acta Univ. Lodz., Folia Bot. 1998; 13: 199-208.

18, Trąba Cz, Ziemińska M. Stan gatunków chwastów segetalnych uważanych za zagrożone w otulinie Roztoczańskiego Parku Narodowego. Acta Univ. Lodz., Folia Bot. 1998; 13: 265-272.

19. Ciosek MT, Skrzyczyńska J. Vicia grandiflora Scop. gatunek ekspansywny (?) w zbiorowiskach roślinnych środkowowschodniej Polski. Fragm. Agron. 2010; 27(3): 44-53.

20. Fijałkowski D. Zbiorowiska roślin synantropijnych miasta Chełma. Annales UMCS, sect. C. 1963; 18(13): 291-235.

21. Fijałkowski D. Zbiorowiska roślin synantropijnych miasta Lublina. Annales UMCS, sect. C. 1967; 22(17): 195-233.

22. Trąba Cz, Ziemińska M. Zbiorowiska chwastów na polach uprawnych w okolicach Zamościa. Cz. I. Zbiorowiska na rędzinach. Annales UMCS, sect. E. 1994; 49(14): 89-98.

23. Kornaś J. Zbiorowiska roślinne Jury Krakowskiej. I. Zespoły pól uprawnych. Acta Soc. Bot. Pol. 1950; 20(2): 361-438. 
24. Wnuk Z. Zbiorowiska chwastów segetalnych Pasma Przedborsko-Małogoskiego i przyległych terenów. Cz. 2. Zbiorowiska zbożowe i ścierniskowe. Corn and stubble-field communities. Acta Univ. Lodz., Zesz. Nauk. Uniw. Łódzkiego. 1976; 14: 123-177.

25. Wnuk Z. Caucalido-Scandicetum R. Tx. 1937 (zespół Caucalis daucoides - Scandix pecten-veneris, Caucalo-Scandicetum Libbert 1930) w Polsce. Acta Univ. Lodz. Folia Bot. 1989; 6: 101-121.

26. Si c ińs k i J T. Zbiorowiska segetalne Kotliny Szczercowskiej (Widawskiej). Acta Agrobot. 1974; 27(2): 5-94.

27. Siciński JT, Sowa R. Zbiorowiska segetalne na glebach rędzinowych w okolicach Sieradza. Acta Agrobot. 1980; 33(2): 269-298.

28. Warcholińska AU. Zbiorowiska chwastów segetalnych Równiny Piotrkowskiej i jej współczesne przemiany w związku z intensyfikacją rolnictwa (Mezoregion Nizin Środkowopolskich). Acta Agrobot. 1974; 27(2): 95-194.

29. Warcholińska AU. Zbiorowiska segetalne Wzgórz Radomszczańskich. Bad. Fizjogr. Pol. Zach., ser. B, Botanika. 1987; 38: 123-153.

30. Ziemińska-Smyk M. Plant communities in arable fields of the Skierbiszów Landscape Park. Acta Agrobot. 2009;62(1): 127-136. http://dx.doi.org/10.5586/aa.2009.015

31. Wiśniewski J. Zespół Vicietum tetraspermae Kruseman et Vlieger 1939 w pow. Łowickim. Zesz. Nauk. Uniw. Łódzkiego. 1970; 36: 53-61.

32. Siciński JT, Sowa R, Warcholińska AU, Wiśniewski J, Wnuk Z. Zróżnicowanie florystyczno-ekologiczne zbiorowisk segetalnych w środkowej Polsce. [W:] R. Sowa, and A. U., Warcholińska (red.) Niektóre aspekty ekologii chwastów segetalnych. Wyd. Uniw. Łódzkiego, Łódź. 1978: 22-40.

33. Kozak M. Zbiorowiska segetalne gminy Rudniki (województwo opolskie). Fragm. Polonica. 2002; 9: 219-272.

\section{Zespoły i zbiorowiska upraw zbożowych Równiny Łukowskiej \\ Cz. II. Zespoły gleb zwięzłych}

\author{
Streszczenie
}

Artykuł ten jest drugą częścią opracowania dotyczącego zbiorowisk segetalnych Równiny Łukowskiej. W pracy przedstawiono charakterystykę fitocenoz wykształcających się w zasiewach zbóż na glebach zwięzłych. W analizowanych siedliskach notowano jedynie Vicietum tetraspermae, nie stwierdzono natomiast płatów Consolido-Brometum ani zbiorowisk ze związku Caucalidion lappulae. Zróżnicowanie florystyczne i ekologiczne Vicietum tetraspermae na badanym terenie realizowało się wyróżnieniem 8 jednostek syntaksonomicznych: 3 podzespołów (Vicietum tetraspermae typicum, V. todontitetosum i V. $t$ consolidetosum) i 6 wariantów w ramach Vicietum tetraspermae typicum. $\mathrm{Na}$ badanym terenie najczęściej notowano płaty Vicietum tetraspermae typicum, rzadziej pozostałe podzespoły. Na szczególną uwagę zasługują ubogie gatunkowo płaty Vicietum tetraspermae typicum zdominowane przez Vicia grandiflora spotykane w okolicach Łukowa jako przejaw degeneracji fitocenoz tego zespołu.

Handling Editor: Elżbieta Weryszko-Chmielewska

This is an Open Access digital version of the article distributed under the terms of the Creative Commons Attribution 3.0 License (creativecommons.org/licenses/by/3.0/), which permits redistribution, commercial and non-commercial, provided that the article is properly cited.

CThe Author(s) 2014 Published by Polish Botanical Society 